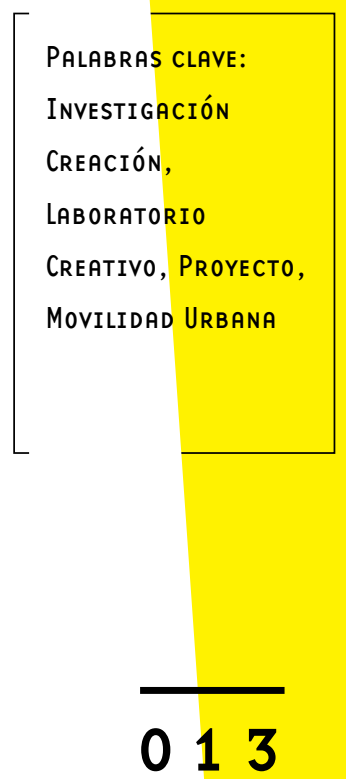

\title{
MOOV LAB - LABORATORIO DE MOVILIDAD
}

https://doi.org/10.53972/RAD.eifd.2018.1.16

CARLOS ANDRÉS PATIÑO YTANIA RECIBIDO 17/08/2018

BENAVIDES, Universidad de Nariño, ACEPTADO 06/09/2018

San Juan de Pasto, Colombia APROBADO 21/10/2018

Resumen: El programa de Diseño Industrial de la Universidad de Nariño incorpora una serie de asignaturas orientadas al componente investigativo, el cual se ha visto afectado por la falta de actualización, concentrándose en una visión positivista que privilegia las estrategias metodológicas tradicionales. Este paradigma es el que ha regido a la hora de plantear los trabajos de grado en la modalidad de proyecto de diseño, situación que ha afectado el componente proyectual de los mismos. A partir de este planteamiento surge el Laboratorio de Movilidad Grupo de Investigación MOV LAB | CORD como una estrategia para la generación de proyectos de investigación-creación orientados al desarrollo de Trabajos de Grado. Esta iniciativa tiene como propósito el planteamiento y desarrollo de dichos proyectos en los espacios académicos de Seminario Trabajo de Grado (noveno semestre) y de Taller de Diseño X | Trabajo de Grado (décimo semestre), mediante la identificación de oportunidades de Diseño en el marco de una problemática macro de alta complejidad o wicked problem (Buchanan. 1992) en este caso referida al fenómeno de la movilidad en la ciudad de San Juan de Pasto. 
¿Sistema integrado de transporte? ¿Sistema?

La ciudad de San Juan de Pasto cuenta con diferentes oportunidades de movilizacion para sus habitantes, aun sin la existencia de un sistema adecuado de proyección y aplicación a la comunidad, la cual involucre y establezca al peatón como principal actor de la ciudad, dando una prioridad en la implementación de nuevas tecnologías y pedagogias que favorezcan a la recuperación y conservación del medio ambiente.

Como estudiantes activos en procesos extracurriculares que han buscado sobresaltar los nuevos métodos y procesos de creación ante una problemática social y enfocada en el entendimiento de nuevos procesos de desarrollo social actuando el diseño como un factor influyente frente al proceso de crecimiento y desarrollo de la ciudad de Pasto, generando una oportunidad donde estudiantes del programa de diseño pueden llevar a cabo procesos de investigación y proyeccion de soluciones que generen beneficios al sistema de movilidad e interacción social de la ciudad, que se ha visto afectada por diversos factores como mala administración y poca participación de sectores que logren aportar soluciones reales y adecuadas al estado actual de la ciudad, teniendo como futuro objetivo la ampliación de estas propuestas como modelos de progreso aplicables a diferentes regiones del territorio nacional.

\section{¿Investigar o crear?}

El programa de Diseño Industrial de la Universidad de Nariño dentro de su plan de estudios incorpora una serie de asignaturas que articulan los procesos de aprendizaje orientados al componente investigativo, que proyectado al final de la carrera tiene como propósito principal apoyar el desarrollo de trabajos de grado presentados por estudiantes. Situación general que se identifica en la mayoría 
de programas académicos correspondientes a Diseño Industrial en Colombia (Calvache. 2018), en el caso de la Universidad de Nariño el desarrollo de los trabajos de grado se ha visto afectado por la falta de actualización del componente investigativo, concentrando la misma a una visión desde el paradigma positivista que de alguna manera privilegia las estrategias metodológicas tradicionales. Por esta razón, ha sido esta la estructura lineal y poco incierta de las academias de diseño que ha regido a la hora de plantear trabajos de grado en la modalidad de proyecto de diseño, situación que ha afectado el factor de tener un componente proyectual y visión de desarrollo de los mismos.

El grupo de investigación cord preocupado por esta situación busca crear el laboratorio de movilidad MOV-LAB, con el fin de contribuir a una mejor inserción de componente investigativo dentro de la academia en el programa de diseño industrial, implementando como metodología, la investigación-creación, apropiándose así los estudiantes de esta, y mejorando el desarrollo de trabajos de grado como proceso de disciplina creativa, además ser contemplados como productos académicos enmarcados en la dinámica planteada por la Mesa de Artes, Arquitectura y Diseño de Colciencias, que ha abierto un espacio importante en términos de la evaluación de productos para las disciplinas creativas resultado de procesos inscritos en la investigación-creación como estrategia por excelencia para la generación de nuevo conocimiento (Colciencias. 2017).

\section{Metodología}

La metodología implementada es investigacion-creacion donde se define con tres pasos el desarrollo de la investigación en las disciplinas creativas que le permite al investigador estructurar su proceso investigativo:

1. La iteración: acto de exploración repetitiva que, al igual que la investigación tradicional requiere de recursos humanos y económicos para llevar a cabo su experimentación.

2. La exploración rigurosa o disciplinada: estructura de pasos exploratorios basados en la intuición, con el propósito de entender el mundo y transformarlo.

3. El lenguaje plástico y sensorial: se utilizan otros métodos de expresión como la imagen, el video y el sonido, cumpliendo la misma función del lenguaje escrito. 


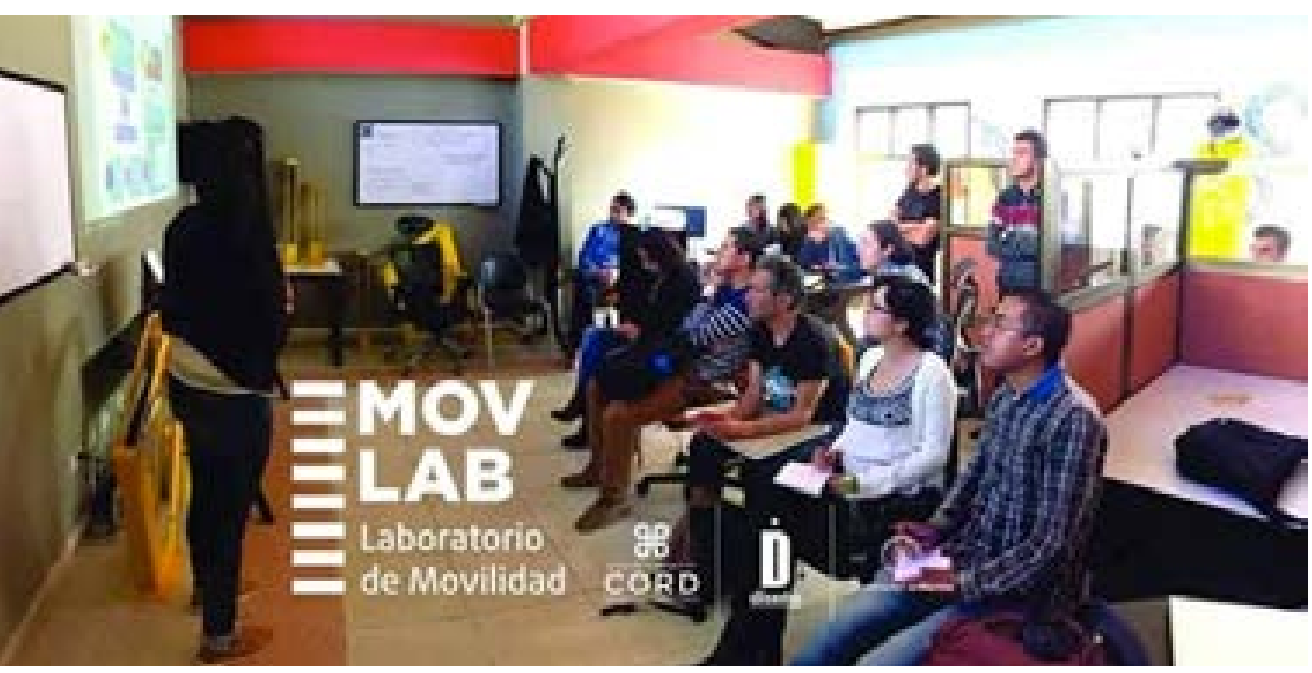

En seguida tres componentes que generen una estructura general que pueden suceder secuencias o simultáneamente en un orden distinto al expuesto aquí:

1. Contextualización: el creador conoce la realidad a investigar y determina tiempo y espacio.

2. Sensación detonante: el creador interpreta la información del contexto, le da sentido y reflexiona sobre el entendimiento explícito en la acción. es un momento de comprensión, critica y reestructuración.

3. Conformación platica: es cuando la investigación toma una forma y lenguaje específico para mostrar el resultado, es donde el creador le da forma a sus ideas y concreta el producto platico sensorial. (Ballesteros y Beltrán. 2018).

\section{¿Diseño y sociedad?}

Dentro de todas las posibilidades existentes dentro de un proceso de investigación existe la variable de crear mediante los mismos pasos de una investigaron tradicional, lo cual nos da a entender que no son tan distintos los procesos pero que la idea de generar procesos no verticales sino horizontales y abiertos a nuevos contextos, conceptos e ideas en general nos genera una amplia posibilidad de entender un solo concepto y expandirlo de tal manera que se despliegue una lista de nuevas oportunidades de diseño, siendo este el caso de los participantes de este laboratorio de investigación en el cual cada integrante presenta desde su experiencia una posibilidad de mejora y desarrollo de su entorno. 


\section{Referencias}

COLCIENCIAS. (2017). Modelo de medición de grupos de investigación, desarrollo tecnológico o de innovación y de reconocimiento de investigadores del Sistema Nacional de Ciencia, Tecnología e Innovación. Bogotá: Colciencias.

BUCHANAN, R. (1992). Wicked Problems in Design Thinking. Design Issues, 8(2), pp. 5-21. Recuperado de: http://www.jstor.org/ stable/1511637?seq=1\#page_scan_tab_contents

BALLESTEROS, M. BELTRÁN, E. (2018). ¿Investigar Creando?: una guía para la investigación-creación en la académica. Bogotá: Universidad El Bosque, Facultad de Creación y Comunicación.

Sobre los autores
Elizabeth Polo Jefe del Departamento de Diseño de la Universidad de Nariño de la ciudad de San Juan de Pasto. Ha desarrollado espacios donde se ha mostrado un interés sobre los nuevos procesos de investigación y desarrollo de tecnologías dentro del programa de diseño para fortalecer aspectos principales dentro del comportamiento social, de tal manera se logra generar una estructura que posibilite a los estudiantes generar nuevos procesos de creación correspondientes a la actualidad mediática en la cual nos desarrollamos de manera inconsciente pero aceptada. 


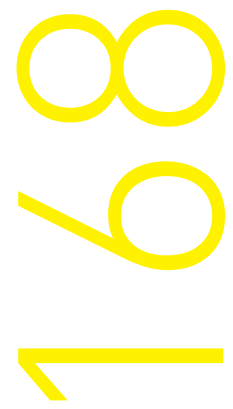

